\title{
Prospective multi-center dosimetry study of low-dose lodine-125 prostate brachytherapy performed after transurethral resection
}

\author{
Carl Salembier, MD', Alex Rijnders', Ann Henry, MD², Peter Niehoff, MD³, Frank André Siebert, PhD', Peter Hoskin, PhD ${ }^{5}$ \\ IRadiation Oncology Department, Europe Hospitals Brussels, Belgium, 2St James' Institute of Oncology, Leeds, United Kingdom, ${ }^{3}$ Radiation \\ Department, Municipal Hospital - University Hospital of Witten-Herdecke, Cologne, Germany, ${ }^{4} U H-S H$ Campus Kiel Klinik für Strahlentherapie \\ und Radiooncologie, Kiel, Germany, ${ }^{5}$ Mount Vernon Cancer Centre Northwood, United Kingdom
}

\begin{abstract}
Purpose: To evaluate in a multicenter setting the ability of centers to perform pre-implant permanent prostate brachytherapy planning, fulfilling dosimetric goals and constraints based on the Groupe de Curiethérapie-European Society for Radiotherapy and Oncology guidelines in the setting of implantation after prior prostate transurethral resection (TURP).

Material and methods: A reference transrectal ultrasound image set of the prostate gland from a patient who had undergone TURP was used. Contouring of the prostate, clinical target volume and organs at risk was performed by the coordinating center. Goals and constraints regarding the dosimetry were defined.

Results: Seventeen of twenty-five centers invited to participate were able to import the Digital Imaging and Communications in Medicine-images into their planning computer and plan the implant using the defined guidelines. All centers were able to plan treatment, and achieve the recommended objectives and constraints. However, sector analysis has shown a risk of under-dosage in the anterior part of the prostate.

Conclusions: Correct pre-implantation planning with adherence to protocol guidelines and in compliance with defined dosimetric constraints seems feasible in a post-TURP setting, at least on a theoretical basis. A prospective study evaluating the outcome of prostate brachytherapy performed after TURP can therefore be undertaken with an expectation of a correct dosimetry in the multicenter setting.

Key words: brachytherapy, permanent seed implant, prostate cancer, transurethral resection, TURP.

\section{Purpose}

Low dose rate I-125 seed brachytherapy is an established treatment for localized low risk prostate cancer. Both the American Brachytherapy Society (ABS) recommendations on permanent seed implant [1,2] and the Groupe de Curiethérapie-European Society for Radiotherapy and Oncology (GEC-ESTRO) guidelines [3] consider prior transurethral resection of the prostate (TURP), a relative contra-indication for prostate permanent seed brachytherapy. In many experienced brachytherapy centers, a history of endoscopic resection of the prostate remains an absolute contra-indication to prostate brachytherapy which is reflected in small number of publications in this area. These recommendations were principally based on an early report from the Seattle group [4], describing their initial experience, and reporting a major risk of significant toxicity and primarily urinary incontinence in brachytherapy patients who had undergone prior TURP. However, this data was based on a relatively small cohort of patients treated with early dosimetry planning sys- tems, and homogeneous loading of the radioactive isotopes, when imaging and dosimetry was not well developed. With more extensive experience in the field of low dose rate (LDR) prostate brachytherapy, optimization of imaging techniques and improved loading and dosimetry techniques, the complication rate in this group of patients has considerably decreased. More recent reports [5-8] dealing with this specific item suggest that brachytherapy can be safely performed in a TURP patient group on condition that modern imaging, and optimized dosimetry techniques are used. Unfortunately, experience remains limited; reports are few and deal with small patient groups. Against this background of early reports in the literature and further personal experience we have developed a protocol for target and organ at risk contouring, definition of CTV and dosimetric parameters, based on the GEC-ESTRO guidelines for prostate seed implantation [9], but with some specific adaptation for the post-TURP situation. Pilot data from the use of this protocol in a small cohort of patients has been presented [8], and a larger prospective cohort study is planned. As part of the 
development of this, a planning exercise to assess the feasibility of adopting the planning protocol in a multicenter setting has been undertaken. Due to the multitude of different implantation techniques available in this multi-center environment, the variability between institutes will be evaluated. The proposed planning protocol will be considered as applicable if the qualitative and quantitative evaluation shows a fulfilling of the stated requirements by the majority of the participating centers.

\section{Material and methods}

Twenty-five prostate brachytherapy centers with extensive experience in I-125 monotherapy were invited to participate in the study. Each center received the data set containing a transrectal ultrasound image set with prostate, CTV, urethral defect and rectum delineated. The clinical history which accompanied the image set was of a patient with a previous history of a transurethral resection of the prostate for obstructive symptoms having been diagnosed with prostate cancer Gleason score $3+3$, and stage pT1a with a rising PSA on active surveillance who had elected to undergo I-125 seed brachytherapy.

Target and organ at risk contouring, definition of CTV and dosimetric parameters were performed according to the following guidelines: clinical target volume (CTV) was defined by the visible contour of the prostate expanded with a threedimensional volume expansion of $3 \mathrm{~mm}$ constrained to the anterior rectal wall, bladder neck and the urethral defect. The urethral defect was excluded from the prostate contour, and contoured entirely taking into account the eventual anterior path often seen after this kind of intervention (Figs. 1 and 2). The prescribed dose to the $100 \%$ isodose was $145 \mathrm{~Gy}$ with the following requirements: 1 ) the $\mathrm{V}_{100}$ (the percentage of the CTV that receives the prescribed dose) must be at least $95 \%\left(V_{100} \geq 95 \%\right.$ of $\left.\left.C T V\right) ; 2\right)$ the $D_{90}$ (the dose that covers $90 \%$ volume of the CTV) will be larger than the prescription dose $\left(D_{90}>100 \%\right.$ of prescription dose); 3$)$ the $V_{150}$ (the percentage of the CTV that receives $150 \%$ of the prescription dose), should be equal to or less than $70 \%$.

All these parameters are in line with ESTRO/EAU/ EORTC recommendations on prostate brachytherapy [9], except for the $V_{150}$. The higher $V_{150}$-value has been defined, given the specific post-TURP situation. Effectively, as noted before, the urethral defect was excluded from the prostate contour. This directly influences the percentage of the CTV that receives doses higher than the prescription dose. In addition, specific constraints were defined for organs at risk. The rectal $\mathrm{D}_{2 \mathrm{cc}}$ was to be limited to $<145 \mathrm{~Gy}$, and $\mathrm{D}_{0.1}$ $<200 \mathrm{~Gy}$; urethral doses (defined by the TURP defect) were to be limited to $D_{\mathrm{u} 10}<150 \%$, and $D_{\mathrm{u} 30}<130 \%$ of the prescription dose. The prostate volume was $17.82 \mathrm{cc}$, and the CTV was $23.50 \mathrm{cc}$. The volume of the urethral defect was $2.52 \mathrm{cc}$.

Each participating center was asked to perform implant dosimetry using seed strength as routinely used in their department on the data set provided, meeting the proposed dosimetric constraints. Responses were obtained from seventeen of the twenty-five investigators invited to participate. All plans were generated using Variseed ${ }^{\circledR}$ (Varian Medical Systems, Palo Alto, CA, USA). Central review of the plans was performed at the Europe Hospitals Brussels, Belgium. This included evaluation of the number of needles, the seed activity, the number of seeds, total activity, target and organs at risk parameters, and additionally sector analysis. Cumulative dose volume histograms (DVH's) were determined for each received plan. The prostate dosimetric evaluation was defined by the volume of the gland receiving $100 \%, 150 \%$ and $200 \%\left(\mathrm{~V}_{\mathrm{p} 100}, \mathrm{~V}_{\mathrm{p} 150}\right.$ and $\left.\mathrm{V}_{\mathrm{p} 200}\right)$ of the prescription dose, and the minimum dose received by $90 \%$ of the prostate gland $\left(\mathrm{D}_{\mathrm{p} 90}\right)$. The CTV dosimetric evaluation was defined by the volume of the CTV receiving $100 \%$ and $150 \%\left(\mathrm{~V}_{100}\right.$ and $\left.\mathrm{V}_{150}\right)$ of the prescription dose, and the minimum dose received by $90 \%$ of the CTV $\left(\mathrm{D}_{90}\right)$. Urethral dosimetry was defined in terms of the minimum administered dose received by $30 \%$ and $10 \%\left(D_{\mathrm{u} 30}\right.$ and $\left.\mathrm{D}_{\mathrm{u} 10}\right)$ of the urethral defect. Finally, also a sector analysis was performed.

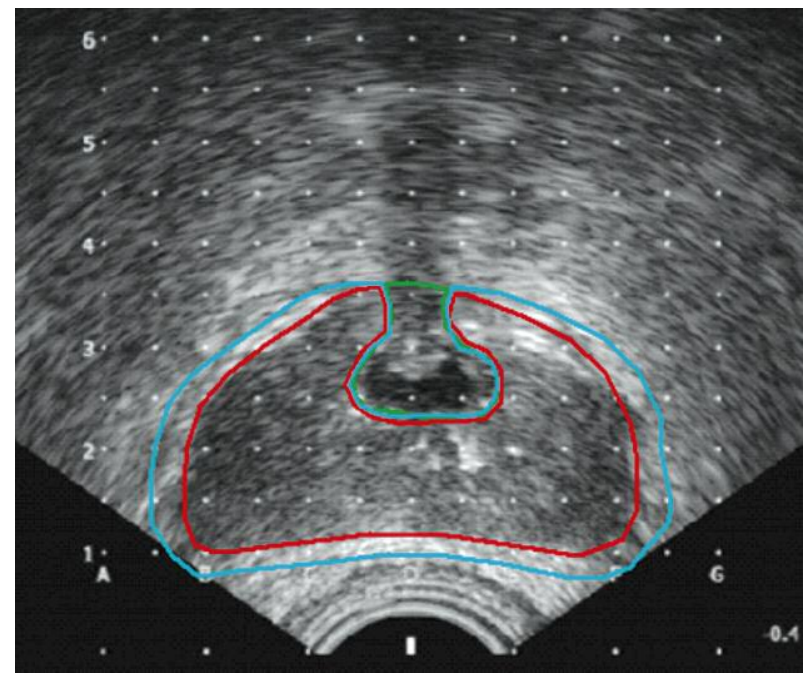

Fig. 2. Expansion from prostate to CTV (= PTV) for prostate brachytherapy after transurethral resection of the prostate

(TURP)
Fig. 1. Contouring of prostate and urethral defect for prostate brachytherapy after transurethral resection of

the prostate (TURP)

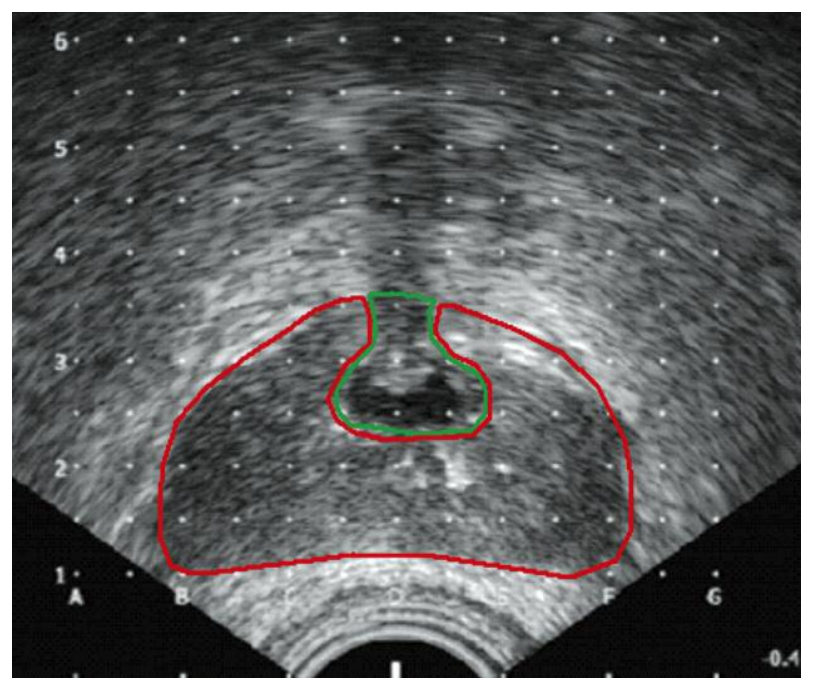


Table 1. Brachytherapy parameters: the seed activity, the number of seeds, the number of needles and the activity of the whole implant by each participating center

\begin{tabular}{|c|c|c|c|c|c|c|}
\hline Center & $\begin{array}{l}\text { Seed activity } \\
\text { (U/seed) }\end{array}$ & $\begin{array}{l}\text { Seed activity } \\
(\mathrm{mCi} / \text { seed })\end{array}$ & $\begin{array}{l}\text { Number } \\
\text { of seeds }\end{array}$ & $\begin{array}{l}\text { Number } \\
\text { of needles }\end{array}$ & $\begin{array}{c}\text { Activity } \\
\text { of the implant } \\
\text { (U) }\end{array}$ & $\begin{array}{l}\text { Activity } \\
\text { of the implant } \\
(\mathrm{mCi})\end{array}$ \\
\hline 1 & 0.635 & 0.500 & 46 & 22 & 29 & 23 \\
\hline 2 & 0.538 & 0.424 & 50 & 18 & 27 & 21 \\
\hline 3 & 0.458 & 0.361 & 60 & 21 & 27 & 22 \\
\hline 4 & 0.470 & 0.370 & 56 & 20 & 26 & 21 \\
\hline 5 & 0.508 & 0.400 & 58 & 19 & 29 & 23 \\
\hline 6 & 0.508 & 0.400 & 52 & 16 & 26 & 21 \\
\hline 7 & 0.613 & 0.483 & 44 & 14 & 27 & 21 \\
\hline 8 & 0.597 & 0.470 & 49 & 13 & 29 & 23 \\
\hline 9 & 0.538 & 0.424 & 63 & 21 & 34 & 27 \\
\hline 10 & 0.508 & 0.400 & 54 & 17 & 27 & 22 \\
\hline 11 & 0.559 & 0.440 & 46 & 15 & 26 & 20 \\
\hline 12 & 0.571 & 0.450 & 49 & 19 & 28 & 22 \\
\hline 13 & 1.000 & 0.787 & 27 & 10 & 27 & 21 \\
\hline 14 & 0.491 & 0.387 & 53 & 19 & 26 & 20 \\
\hline 15 & 0.491 & 0.387 & 58 & 25 & 28 & 22 \\
\hline 16 & 0.491 & 0.387 & 58 & 20 & 28 & 22 \\
\hline 17 & 0.458 & 0.361 & 58 & 23 & 27 & 21 \\
\hline
\end{tabular}

This analysis was done using commercially available option in the Variseed ${ }^{\circledR}$ system. Sector Analysis ${ }^{\circledR}$ tool from Variseed $8.0^{\circledR}$ divides the prostate automatically into up to 12 sectors, and provides dose parameters for each one. The prostate gland is divided into two or three regions in the cranio-caudal direction (base and apex in case of a small prostate, base, mid-gland, and apex in case of a larger prostate), and four regions on each transverse slide (anterior, posterior, left and right). This gives eight or twelve sectors. Given the rather small volume of the prostate used in this exercise, only eight different segments were defined and analyzed.

\section{Results}

Seventeen different dosimetric plans were obtained. The number of needles and seeds, the seed strength, and the activity of the implant showed considerable variation as detailed in Table 1 . The mean number of needles used was 18 , with a minimum of 10 needles and a maximum of 25 . The number of seeds used varied between 27 and 63 , with a mean of 52 seeds. Eight out of 17 centers applied a classical seed spacing with regular $1 \mathrm{~cm}$ seed center-tocenter in the majority of their needles, whereas 9 centers used specially loaded needles that differed by having extra spacing between seeds. The mean seed strength used was $0.486 \mathrm{U}$ (range $0.458-1.000 \mathrm{U}$ ) or $0.383 \mathrm{mCi}$ (range 0.361 $0.787 \mathrm{mCi})$. The mean activity of the total implant was $28 \mathrm{U}$ (range 26-34 U) or $22 \mathrm{mCi}$ (range 20-27 mCi).

All centers fulfilled the requested dosimetry constraints for the prostate volume. The mean $\mathrm{V}_{\mathrm{p} 100}$ was $98 \%$ (range
97-100\%), the mean $\mathrm{D}_{\mathrm{p} 90}$ was $187 \mathrm{~Gy}$ (range 159-219 Gy), the mean $\mathrm{V}_{\mathrm{p} 150}$ was $73 \%$ (range $42-90 \%$ ), and the mean $\mathrm{V}_{\mathrm{p} 200}$ was $35 \%$ (range $18-56 \%$ ). Table 2 shows the dosimetric analysis for the prostate and the CTV for each dosimetry data set. All but two centers fulfilled the CTV dosimetric constraints, and these two centers deviated from the requirements by only a small amount, which could have been corrected by movement of one or two seeds by a few millimeters. The mean $\mathrm{V}_{100}$ for the CTV was $96 \%$ (range $94-99 \%$ ), mean $\mathrm{V}_{150}$ was $62 \%$ (range $41-75 \%$ ) and mean $\mathrm{D}_{90}$ was $171 \mathrm{~Gy}$ (range 157-186 Gy).

In terms of urethral dosimetry (Table 3), all but two institutions were able to fulfill the protocol requirements. These two centers exceeded the $\mathrm{D}_{\mathrm{u} 30}$ constraint achieving $133 \%$, and $134 \%$ in contrast to the protocol constraint of $\leq 130 \%$ ). The mean $\mathrm{D}_{\mathrm{u} 30}$ was $118 \%$ (range $99-134 \%$ ) and the mean $\mathrm{D}_{\mathrm{u} 10}$ was $124 \%$ (range $104-148 \%$ ). All centers achieved the rectal dose constraints. Sector analysis was performed for all plans. Given the small prostatic volume, eight segments were defined as shown in Figure 3. The typical post-TURP defect results in very small anterior segments. For each segment, $\mathrm{D}_{90}$ and $\mathrm{V}_{100}$ were calculated as shown in Table 4. Three centers showed perfect coverage for $D_{90}$ and $V_{100}$ for all defined segments. Four centers had a clear under-dosage in the basal anterior segments and fourteen centers had an under-dosage in the apical anterior segments.

\section{Discussion}

The aim of this multi-center prospective analysis was to assess the feasibility of a modified dosimetry guideline 
Table 2. Brachytherapy parameters regarding the prostate $(p)$ and the clinical target volume (ctv) by each participating center

\begin{tabular}{llllllll} 
Center & $\begin{array}{c}\mathrm{V}_{\mathrm{p100}} \\
(\%)\end{array}$ & $\begin{array}{c}\mathrm{V}_{\mathrm{p150}} \\
(\%)\end{array}$ & $\begin{array}{c}\mathrm{V}_{\mathrm{p200}} \\
(\%)\end{array}$ & $\begin{array}{c}\mathrm{D}_{\mathrm{p} 90} \\
(\mathrm{~Gy})\end{array}$ & $\begin{array}{c}\mathrm{V}_{\text {ctv100 }} \\
(\%)\end{array}$ & $\begin{array}{c}\mathrm{V}_{\text {ctv150 }} \\
(\%)\end{array}$ & $\begin{array}{c}\mathrm{D}_{\text {ctv90 }} \\
(\mathrm{Gy})\end{array}$ \\
\hline 1 & 99 & 90 & 53 & 219 & 99 & 75 & 186 \\
\hline 2 & 99 & 74 & 32 & 185 & 98 & 68 & 175 \\
\hline 3 & 99 & 66 & 26 & 175 & 96 & 60 & 167 \\
\hline 4 & 97 & 70 & 25 & 183 & 96 & 64 & 169 \\
\hline 5 & 98 & 74 & 31 & 182 & 96 & 66 & 170 \\
\hline 6 & 100 & 59 & 22 & 181 & 99 & 55 & 176 \\
\hline 7 & 97 & 64 & 29 & 178 & 97 & 59 & 167 \\
\hline 8 & 98 & 73 & 37 & 184 & 97 & 70 & 176 \\
\hline 9 & 100 & 82 & 37 & 199 & 99 & 75 & 185 \\
\hline 10 & 97 & 81 & 35 & 196 & 96 & 74 & 177 \\
\hline 11 & 98 & 75 & 28 & 191 & 96 & 66 & 173 \\
\hline 12 & 97 & 83 & 56 & 191 & 94 & 71 & 160 \\
\hline 13 & 97 & 69 & 35 & 177 & 94 & 62 & 162 \\
\hline 14 & 98 & 42 & 18 & 159 & 96 & 41 & 157 \\
\hline 15 & 99 & 82 & 46 & 199 & 97 & 73 & 180 \\
\hline 16 & 99 & 83 & 47 & 199 & 96 & 73 & 171 \\
\hline 17 & 97 & 73 & 37 & 180 & 95 & 64 & 164 \\
\hline
\end{tabular}

Table 3. Brachytherapy parameters regarding the urethral defect: $D_{\mathrm{u} 10}$ and $\mathrm{D}_{\mathrm{u} 30}$ by each participating center

\begin{tabular}{|c|c|c|}
\hline Center & $\mathrm{D}_{\mathrm{u} 30}(\%)$ & $\mathrm{D}_{\mathrm{u} 10}(\%)$ \\
\hline 1 & 133 & 148 \\
\hline 2 & 113 & 123 \\
\hline 3 & 122 & 133 \\
\hline 4 & 112 & 125 \\
\hline 5 & 120 & 139 \\
\hline 6 & 117 & 126 \\
\hline 7 & 114 & 126 \\
\hline 8 & 113 & 125 \\
\hline 9 & 134 & 146 \\
\hline 10 & 124 & 137 \\
\hline 11 & 118 & 130 \\
\hline 12 & 127 & 148 \\
\hline 13 & 110 & 122 \\
\hline 14 & 99 & 104 \\
\hline 15 & 114 & 127 \\
\hline 16 & 128 & 145 \\
\hline 17 & 112 & 131 \\
\hline
\end{tabular}

for prostate brachytherapy in patients who have had a previous TURP leaving a residual defect. This guideline defines the outlining of the prostate, and urethral defect with specific adaptations for volume-expansion to obtain a work- able CTV with recommendations for target and organs at risk dose constraints. This has now been tested in a multicenter setting. In the absence of a TURP cavity urinary incontinence, following I- 125 seed brachytherapy has been related to urethral dose and pre-implant urinary symptom score (IPSS). In a detailed study of I-125 monotherapy delivering $145 \mathrm{~Gy}$, the mean urethral $\mathrm{D}_{10}$ in patients having no urinary toxicity was $314 \mathrm{~Gy}$ compared to $394 \mathrm{~Gy}$ in those with any grade of incontinence; the incidence doubled in patients who received a dose of $>450$ Gy [10]. Current GECESTRO guidelines recommend that the urethral $D_{10}$ is kept below $150 \%$ i.e. $217.5 \mathrm{~Gy}$, and this dose constraint is used in the protocol described here. There is no published data comparing tolerance in the urethra following TURP compared to that before. The overall result of this prospective multi-center evaluation showed that it was feasible to perform dosimetry compliant with the protocol with only small deviations in two instances for the CTV and urethral doses. These results were obtained despite different institutions employing varying seed strengths, loading patterns and numbers of needles. However, sector analysis showed that an under-dosage of the anterior segments (basal and/or apical) of the prostate can occur despite remaining within the defined global dose recommendations for the CTV.

This is the first multicenter evaluation of prostate brachytherapy dosimetry in the post-TURP situation with defined achievable dose constraints. Although this evaluation is based on only one example, this prostate patient is considered a typical case in this setting with a significant urethral defect in relation to the overall prostate volume. One other study [11] has addressed the issue of implant quality after TURP, and showed that patients with substantial 

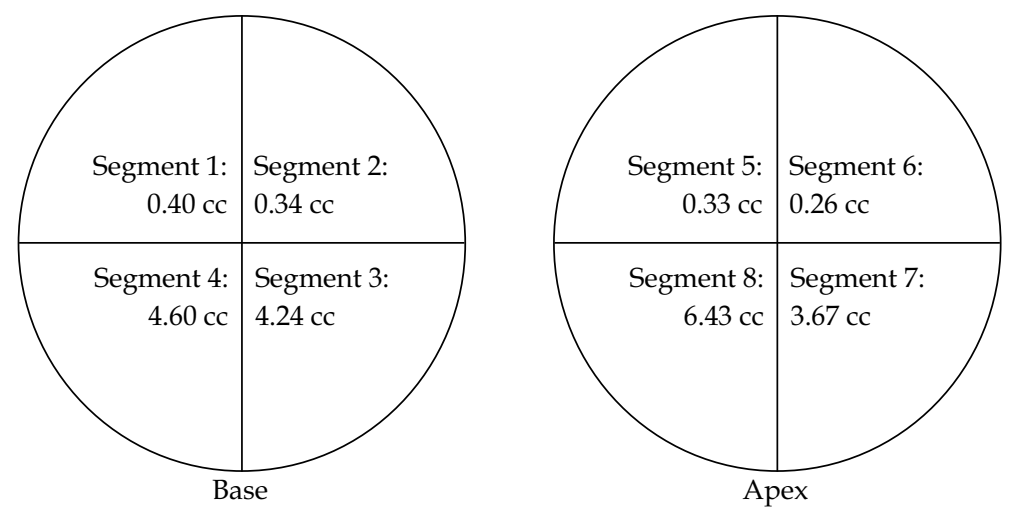

Fig. 3. Sector analysis with segment volumes

Table 4. Dosimetric parameters per segment for all participants $\left(V_{100}\right.$ and $D_{90}$ for each specific segment)

\begin{tabular}{|c|c|c|c|c|c|c|c|c|c|}
\hline & & Segment 1 & Segment 2 & Segment 3 & Segment 4 & Segment 5 & Segment 6 & Segment 7 & Segment 8 \\
\hline \multirow[t]{2}{*}{ Center 1} & $V_{100}(\%)$ & 100 & 100 & 100 & 100 & 100 & 100 & 100 & 100 \\
\hline & $\mathrm{D}_{90}$ (Gy) & 178 & 179 & 202 & 200 & 167 & 171 & 211 & 212 \\
\hline \multirow[t]{2}{*}{ Center 2} & $V_{100}(\%)$ & 100 & 100 & 100 & 100 & 98 & 100 & 100 & 100 \\
\hline & $\mathrm{D}_{90}(\mathrm{~Gy})$ & 185 & 205 & 190 & 180 & 172 & 163 & 185 & 179 \\
\hline \multirow[t]{2}{*}{ Center 3} & $\mathrm{~V}_{100}(\%)$ & 96 & 100 & 99 & 99 & 100 & 95 & 99 & 99 \\
\hline & $\mathrm{D}_{90}$ (Gy) & 156 & 170 & 195 & 183 & 163 & 163 & 189 & 194 \\
\hline \multirow[t]{2}{*}{ Center 4} & $V_{100}(\%)$ & 100 & 100 & 100 & 100 & 73 & 62 & 96 & 99 \\
\hline & $\mathrm{D}_{90}$ (Gy) & 183 & 210 & 215 & 218 & 119 & 111 & 171 & 195 \\
\hline \multirow[t]{2}{*}{ Center 5} & $V_{100}(\%)$ & 98 & 100 & 100 & 100 & 76 & 67 & 97 & 98 \\
\hline & $\mathrm{D}_{90}$ (Gy) & 179 & 226 & 211 & 202 & 120 & 118 & 179 & 182 \\
\hline \multirow[t]{2}{*}{ Center 6} & $V_{100}(\%)$ & 100 & 100 & 100 & 100 & 78 & 74 & 99 & 100 \\
\hline & $\mathrm{D}_{90}$ (Gy) & 177 & 189 & 204 & 226 & 120 & 126 & 190 & 211 \\
\hline \multirow[t]{2}{*}{ Center 7} & $V_{100}(\%)$ & 93 & 97 & 100 & 100 & 62 & 64 & 98 & 99 \\
\hline & $\mathrm{D}_{90}$ (Gy) & 155 & 160 & 200 & 187 & 114 & 116 & 187 & 185 \\
\hline \multirow[t]{2}{*}{ Center 8} & $\mathrm{~V}_{100}(\%)$ & 100 & 100 & 100 & 100 & 73 & 71 & 100 & 100 \\
\hline & $\mathrm{D}_{90}$ (Gy) & 189 & 181 & 232 & 236 & 126 & 125 & 219 & 224 \\
\hline \multirow[t]{2}{*}{ Center 9} & $\mathrm{~V}_{100}(\%)$ & 98 & 100 & 100 & 100 & 89 & 88 & 98 & 98 \\
\hline & $\mathrm{D}_{90}$ (Gy) & 160 & 178 & 179 & 183 & 139 & 148 & 171 & 175 \\
\hline \multirow[t]{2}{*}{ Center 10} & $\mathrm{~V}_{100}(\%)$ & 93 & 100 & 100 & 100 & 71 & 69 & 98 & 99 \\
\hline & $\mathrm{D}_{90}(\mathrm{~Gy})$ & 151 & 196 & 195 & 188 & 120 & 119 & 185 & 186 \\
\hline \multirow[t]{2}{*}{ Center 11} & $\mathrm{~V}_{100}(\%)$ & 93 & 97 & 99 & 99 & 87 & 76 & 98 & 99 \\
\hline & $\mathrm{D}_{90}(\mathrm{~Gy})$ & 146 & 161 & 168 & 164 & 142 & 137 & 157 & 160 \\
\hline \multirow[t]{2}{*}{ Center 12} & $\mathrm{~V}_{100}(\%)$ & 93 & 100 & 100 & 100 & 78 & 74 & 99 & 100 \\
\hline & $\mathrm{D}_{90}$ (Gy) & 154 & 191 & 223 & 213 & 127 & 124 & 191 & 197 \\
\hline \multirow[t]{2}{*}{ Center 13} & $\mathrm{~V}_{100}(\%)$ & 95 & 94 & 99 & 100 & 69 & 62 & 99 & 99 \\
\hline & $\mathrm{D}_{90}$ (Gy) & 157 & 150 & 190 & 214 & 121 & 115 & 203 & 218 \\
\hline \multirow[t]{2}{*}{ Center 14} & $V_{100}(\%)$ & 88 & 91 & 100 & 100 & 87 & 83 & 99 & 100 \\
\hline & $\mathrm{D}_{90}$ (Gy) & 142 & 148 & 190 & 184 & 142 & 138 & 178 & 182 \\
\hline \multirow[t]{2}{*}{ Center 15} & $V_{100}(\%)$ & 88 & 85 & 100 & 100 & 68 & 55 & 98 & 99 \\
\hline & $\mathrm{D}_{90}$ (Gy) & 136 & 140 & 204 & 197 & 117 & 113 & 172 & 177 \\
\hline \multirow[t]{2}{*}{ Center 16} & $V_{100}(\%)$ & 93 & 91 & 100 & 100 & 73 & 62 & 98 & 99 \\
\hline & $\mathrm{D}_{90}$ (Gy) & 154 & 150 & 196 & 197 & 122 & 115 & 179 & 196 \\
\hline \multirow[t]{2}{*}{ Center 17} & $\mathrm{~V}_{100}(\%)$ & 83 & 94 & 100 & 100 & 76 & 76 & 100 & 100 \\
\hline & $D_{90}(G y)$ & 134 & 158 & 189 & 183 & 132 & 131 & 200 & 210 \\
\hline
\end{tabular}


TURP defects received the same quality of implant as patients with no or small TURP defect. The same study showed an increase in Du30 in patients treated with I-125, if the defect (TURP volume as a percentage of prostate volume) was larger or equal to $20 \%$ compared with no defect, small defect $(<10 \%)$ or medium defect $(10-19 \%)$; the example used in this case and a defect which was $14 \%$ of the total volume. However, it was concluded that it was not possible to compare meaningfully the urethral dose $\left(D_{u 30}\right)$ among the patients with or without TURP defect, because of the heterogeneity of the implant isotopes and treatment strategy.

Contouring and delineation of the prostate capsule and TURP cavity was not included as part of the study. Whilst this does not reflect the real situation where each center has to define all volumes, it was important for the end points in this study to exclude contouring, and delineation bias and focus completely on the dosimetric analysis. Large inter-observer variations are described regarding all different imaging techniques [12-18], even when CT and MRI is used. When TRUS has been used alone, standard deviations varying from $2 \%$ to $13 \%$ of the mean (median: $7 \%$ ) for pre-implant prostate volumes have been described in literature [19]. In addition, important information is obtained during the ultrasound examination which cannot be reproduced by static images which introduces a further variable in contour definition.

The dosimetry results demonstrate rather large range and variation, especially for the $D_{90}$. All centers were asked to perform dosimetry using seed strengths, and implantation technique as routinely used in their department and this will therefore reflect the institutional practice. Real-time intra-operative planning results in even greater individualization of implant geometry, and may increase further this variation. The prospective trial will therefore focus on this item, as well on the impact and evaluation in a post-implant setting.

Sector analysis is normally not used in a pre- or intraoperative setting, but usually in a post-implant setting. However, it may have a role in this specific post-TURP situation. In the post TURP setting there is a fragile balance between the delivery of an adequate dose to all parts of the prostate including the anterior segments, whilst remaining within dose constraints for the urethral defect. This will often depend upon the extent of the urethral defect and the amount of anterior gland which is lost. A number of patients will present for treatment with tumor found in the resected periurethral tissue. An adequate dose must therefore be delivered to the anterior parts of the prostate. Sector analysis has been shown to provide useful additional information in this setting, identifying specific regions which may be under dosed and lead to systematic errors and should be used to optimize implant dosimetry. It provides additional topographic information on dose distribution which enables specific attention to this issue, so that there is sufficient coverage of all parts at risk.

This feasibility study confirmed that in a multicenter setting experienced brachytherapy units can achieve the dose constraints defined in this modified protocol for patients undergoing prostate brachtherapy post TURP. With this reassurance, the UroGEC group of GEC-ESTRO are proceeding with a prospective clinical study in which patients will be treated using the protocol described here with close evaluation of post implant urinary function. It is hoped that the results of this study will provide evidence for future patients who have undergone TURP, to undergo seed brachytherapy with the reassurance that it is a safe and effective treatment for prostate cancer despite previous prostate surgery.

\section{Conflict of interest notification}

Actual or potential conflicts of interest do not exist.

\section{Acknowledgements}

The authors are grateful to all contributing brachytherapists and physicists from the radiation oncology centers of Brussels, Kortrijk, Antwerp, Turnhout and Leuven (Belgium), London, Leeds, Belfast and Edinburgh (Great Britain), Paris (France), Trento (Italy), La Coruna (Spain), Kiel (Germany) and Vienna (Austria).

\section{References}

1. Nag S, Beyer D, Friedland J et al. American Brachytherapy Society (ABS) recommendations for transperineal permanent brachytherapy of prostate cancer. Int J Radiat Oncol Biol Phys 1999; 44: 789-799.

2. Davis BJ, Horwitz EM, Lee WR et al. American Brachytherapy Society consensus guidelines for transrectal ultrasoundguided permanent prostate brachytherapy. Brachytherapy 2012; 11: 6-19.

3. Ash D, Flynn A, Battermann J et al. ESTRO/EAU/EORTC recommendations on permanent seed implantation for localized prostate cancer. Radiother Oncol 2000; 57: 315-321.

4. Blasko JC, Ragde H, Grimm PD. Transperineal ultrasoundguided implantation of the prostate: morbidity and complications. Scand J Urol Nephrol Suppl 1991; 137: 113-118.

5. Wallner K, Lee $H$, Wasserman $S$ et al. Low risk of urinary incontinence following prostate brachytherapy in patients with a prior transurethral prostate resection. Int J Radiat Oncol Biol Phys 1997; 37: 565-569.

6. Stone NN, Ratnow ET, Stock RG. Prior transurethral resection does not increase morbidity following real-time ultrasoundguided prostate seed implantation. Tech Urol 2000; 6: 123-127.

7. Moran JM, Stutz MA, Gurel MH. Prostate brachytherapy can be performed in selected patients after transurethral resection of the prostate. Int J Radiat Oncol Biol Phys 2004; 59: 392-396.

8. Salembier C, Martens G, De Groote P et al. Prior transurethral resection of the prostate should not be a contra-indication for prostate seed brachytherapy. European Multidisciplinary Meeting on Urological Cancers, Barcelona 2009; November 27-29. Abstract P021-60.

9. Salembier C, Lavagnini P, Nickers P et al., on behalf of the PROBATE group of GEC ESTRO. Tumour and target volumes in permanent prostate brachytherapy: A supplement to the ESTRO/EAU/EORTC recommendations on prostate brachytherapy. Radiother Oncol 2007; 83: 3-10.

10. McElveen $\mathrm{T}$, Waterman F, Hayeon $\mathrm{K}$ et al. Factors predicting for urinary incontinence after prostate brachytherapy. Int J Radiat Oncol Biol Phys 2004; 59: 1395-1404.

11. Cesaretti JA, Stone NN, Stock MD. Does prior transurethral resection of the prostate compromise brachytherapy quality: a dosimetry analysis. Int J Radiat Oncol Biol Phys 2004; 60: 648-653.

12. Cazzaniga LF, Marinoni MA, Bossi A et al. Interphysician variability in defining the planning target volume in the irradiation of prostate and seminal vesicles. Radiother Oncol 1998; 47: 293-296.

13. De Brabandere M, Haustermans K, van den Heuvel F et al. Prostate post-implant dosimetry: interobserver variability in 
seed localization, contouring and fusion. Radiother Oncol 2011; 99: 86-87.

14. Fiorino C, Reni M, Bolognesi A et al. Intra- en inter-observer variability in contouring prostate and seminal vesicles: implications for conformal treatment planning. Radiother Oncol 1998; 47: 285-292.

15. Gao Z, Wilkins D, Eapen L et al. A study of prostate delineation referenced against a gold standard created from the visible human data. Radiother Oncol 2007; 85: 239-246.

16. Matzinger O, Poortmans P, Giraud JY et al. Quality assurance in the 22991 EORTC ROG trial in localized prostate cancer: dummy run and individual case review. Radiother Oncol 2009; 90: 285-290.

17. Seddon B, Bidmead M, Wilson J et al. Target volume definition in conformal radiotherapy for prostate cancer: quality assurance in the MRC RT-01 trial. Radiother Oncol 2000; 56: 73-83.

18. Rosewall T, Kong V, Vespirini D et al. Prostate delineation using CT and MRI for radiotherapy patients with bilateral hip protheses. Radiother Oncol 2009; 90: 325-330.

19. Smith S, Wallner K, Merrick G et al. Interpretation of pre-versus postimplant TRUS images. Med Phys 2003; 30: 920-924. 